\title{
HOW DOES BIOLOGICALLY INSPIRED DESIGN COPE WITH MULTI-FUNCTIONALITY?
}

\author{
Svendsen, Nicklas; Lenau, Torben Anker \\ Technical University of Denmark
}

\begin{abstract}
As catalysts for product innovation and product development, different approaches for biologically inspired design (BID) are exciting options. However, while general BID theory require a focus on single functions, real world products are characterized by performing multiple functions. The development of an anterior eye-chamber model is used to showcase the issue.

In a systematic literature review (SLR), state-of-the-art methodologies, methods and tools BID practice are discovered and the current state of multi-functionality in BID are assessed.

The SLR revealed 18 contributions with 8 BID methodologies and 12 stage-specific BID tools (of which $50 \%$ addressed the solution search phase) in addition to 5 papers addressing multi-functionality in BID. At present multi-functionality in BID is only treated in a limited set of papers. While designers interested in BID are advised to discover multi-functional analogies, the present approach to handling multifunctional problems in BID suggest functional decomposition and multiple BID efforts. Therefore, the development of design support for handling multi-functional problems, including tools for problem analysis are needed.
\end{abstract}

Keywords: Bio-inspired design / biomimetics, Design methodology, Design process, Conceptual design, Multi-functionality

Contact:

Svendsen, Nicklas

Technical University of Denmark

Denmark

nwsve@mek.dtu.dk

Cite this article: Svendsen, N., Lenau, T.A. (2019) 'How Does Biologically Inspired Design Cope with MultiFunctionality?', in Proceedings of the 22nd International Conference on Engineering Design (ICED19), Delft, The Netherlands, 5-8 August 2019. DOI:10.1017/dsi.2019.38 


\section{INTRODUCTION}

Despite the fact that most products are characterized by solving a number of functions only few methods for conceptual biological inspired design (BID) support simultaneous handling of more functions. Instead, the methods recommend that solutions are found one function at a time followed by a combination of the partial solutions. However, since natural organisms have survived thanks to a superior fulfilment of a range of functions designers could benefit from being able to handle solution search considering more functions simultaneously. To this end the designers need a methodology supporting the incorporation of multiple functions to develop and launch innovative products. A methodology will include a set of methods and tools addressing different activities in the BID-work. This introduction provides an overview of BID approaches, outline the motivation for studying multifunctionality in BID and illustrate the need for supporting multi-functionality with a practical example. As BID researchers use a broad terminology, the designation 'methodology' will be used to cover BID contributions describing the hole development process (e.g. frameworks, process models, guidelines, etc.). Similarly, the designation 'tools' will be used to cover phase-specific BID support (methods, means, techniques, etc.).

\subsection{Approaches in biologically-inspired design}

In order to develop new and innovative solutions, biology pose an extensive source of knowledge and inspiration to engineering product development - a discipline with many different approaches, amongst others denoted biologically-inspired design, biomimetics, biomimicry, bionics, etc.

ISO 18458 (Biomimetics - Terminology, concepts and methodology) defines the different approaches as follows:

- Bio-inspiration: Creative approach based on the observation of biological systems

- Biomimetics: Interdisciplinary cooperation of biology and technology or other fields of innovation with the goal of solving practical problems through the function analysis of biological systems, their abstraction into models, and the transfer into and application of these models to the solution

- Biomimicry: Philosophy and interdisciplinary design approaches taking nature as a model to meet the challenges of sustainable development

- Bionics: Technical discipline that seeks to replicate, increase, or replace biological functions by their electronic and/or mechanical equivalents

BID as a design activity is carried out by a rather diverse set of professions. Engineers contribute with the formal design methods and theories while biologists with their deep insight into the natural world are often the driving forces in effectuating the knowledge transfer and inventing new bioinspired products, such as the lotus self-cleaning paint (Neinhuis and Barthlott, 1997). Due to the different views of engineers and biologists, approaches and process models for both domains have been proposed. These approaches are most popularly denoted solution-driven BID when the study of a biological phenomenon of interest drives a product development effort and problem-driven BID when a technical functional problem are guiding the product development effort (Helms, Vattam and Goel, 2009).

The starting point of solution-oriented BID is a biological phenomenon of interest, followed by a range of activities leading to an application area for a functional principle inspired by biology. Thereby, the development process of solution-oriented BID takes biologists from biology into the engineering domain. Likewise, the development process of problem-oriented BID leads the designer from the engineering domain, over biology and back to the engineering domain. In practice, many research groups have studied the transition from biology to the engineering domain (occurring for both BID approaches). In this regard, efforts by researchers from the Technical University of Denmark (Lenau, 2009), Virginia Tech (Kennedy, Buikema and Nagel, 2015), the Technical University of Munich (Hashemi Farzaneh, 2016) and Paris Tech (Graeff, Maranzana and Aoussat, 2018) have all pointed towards the importance of incorporating biologists in BID development projects.

\subsection{Why studying multi-functionality in biologically inspired design?}

Problem-oriented BID require that the engineering problem is broken down and reduced to single functions. It is assumed that the solution to the overall engineering problem can be found by using the 
super positioning principle where solutions for each of the required functions are combined. However, it is not given that such a combination is optimal or even good. Natural phenomena on the other hand, have been optimized by evolution through survival of the fittest and for the given context found the best compromise between solving all of the functional challenges. Nature's trial and error strategy has therefore found good solutions to multifunctional problems. It could therefore be attractive to not only search for how single functions are solved in nature, but also look at how the combination of several functions is realized.

An example of how nature finds good solutions to multifunctional problems is the way the nutrition transport system in slime moulds grow (Tero et al., 2010). The slime mould Physarum polycephalum is a one-cell organism which takes up nutrition from the substance it is placed on. Since the nutrition sources are not evenly distributed over the surface, the slime mould need to be economic and build food corridors only to the most valuable food sources. It does so by growing the corridor in all directions from the centre. Once food sources are detected, the relevant food corridors are reinforced while corridors leading to nowhere are broken down and the organic material used elsewhere in the mould. However, the slime mould has at least 3 contradicting challenges: It needs to be economic with the organic material used to make the corridors (save material function), the time for transporting nutrition should be as short as possible (fast transportation function) and finally the system should be resilient to collapsing corridors - there should be alternative routes (resilience function). Manmade computer algorithms are typically only capable of finding optimal solutions for one function at a time, and it is therefore difficult to find the best solution to the combined problem. However, by studying the strategies of the slime mould the best multifunctional solution can be made.

\subsection{Coping with multiple integrated functions developing medical equipment}

To illustrate the need for handling multiple integrated functions in parallel, the development of piece of medical equipment will serve as a case. The work is done in collaboration with the eye department at a university hospital. The case concerns the development of an anterior eye-chamber model capable of simulating the environment of the anterior eye-chamber in order to perform in-vitro corneal transplantations and study post-operative cell behaviour. Long corneal transplant life in ensured by controlling the three essential parameters: Aqueous humor liquid temperature (1), aqueous humor liquid flow rate (2) and intraocular pressure (3). Using the working assumption, that approximating the specifications of these biophysical parameters in the human eye will increase the possibility of corneal transplant life, the following values are the target for the anterior eye-chamber simulator:

- Intraocular pressure: $10-20 \mathrm{mmHg}$

- Aqueous humor liquid flow rate: $3-4 \mu \mathrm{L} / \mathrm{min}$

- Aqueous humor liquid temperature: $35^{\circ} \mathrm{C}$

Using single function search for solutions easily leads to unnecessary complex solution since each function will be handled by separate mechanisms. In practice, flow of aqueous humor liquid (AHL) could be achieved with a peristaltic pump while heating of the AHL could be done with a thermostatic heating element. However, using a peristaltic pump will generate a fluctuating flow and cause alternating high and low pressure of the AHL. Similarly, a fluctuating AHL temperature will be observed if water, stored in a reservoir enclosing the tubing with AHL, is heated with a thermostatic heating element while overheating could be observed locally. Thus, sub functions related to controlling pressure, flow rate and temperature arise from providing simple and straightforward solutions for each of the three main functions of the anterior eye-chamber model. Hence, deriving single-function solutions for each main function rapidly generate a complex product.

\section{METHODS}

State-of-the-art methodology for BID has been examined in order to assess the current state of handling multi-functionality in BID. This has been done in a systematic literature review with following three phases: Planning (1), execution (2) and analysis (3).

\subsection{Systematic literature review planning}

During the planning phase, a systematic literature review protocol was developed in order to specify research questions, to determine articles' selection criteria and qualification criteria used to evaluated 
the found articles in the execution phase and finally put forward which elements should be extracted for analysis. For this particular systematic literature review, the research focus was encapsulated in the following objectives: Identify existing methodologies and phase-specific tools for BID (1) and obtain knowledge on how BID theory copes with multi-functionality (2).

Initially, advice from the articles 2nd author experienced in the BID research field was received, pointing towards +10 high-quality articles in the field, which guided the formulation of keywords. Finally, 4 blocks of keywords were constructed (Block 1 with French and German translations also):

- Block 1: Keywords encapsulating biologically-inspired design approaches and synonyms

- Block 2: Keywords encapsulating 'methodology' and synonyms

- Block 3: Keywords encapsulating 'tool' and synonyms

- Block 4: Keywords encapsulating BID activities and synonyms

Subsequently, the search strategy was determined with focusing on not finding articles describing biomimetic inventions without describing the development process also. Thereby, three different search strings were composed:

1. (English keywords of Block 1 in 'title') AND (Block 2 OR Block 3 OR Block 4 in 'title', 'abstract' or 'keywords') AND NOT ('biomimetic' OR 'bionic')

2. Adjectives of English keywords of Block 1 combined with Block 2 or Block 3 keywords in 'title' - e.g. biomimetic tool*, bio-inspired framework*)

3. (French and German keywords of Block 1 in 'title')

In order to ensure the search strings' validity, these was verified by cross-checking that articles recommended by the specialist was 'captured'.

\subsection{Systematic literature review execution}

In order to execute the systematic literature review Scopus and Design Society's electronic databases were used to retrieve articles. However, as a test search executing the three search strings revealed a total of 2.308 articles, filtering with regards to subject area was done initially in order to increase relevance of the literature base. To ensure inclusion of the relevant subject areas, top-20 most cited articles from the subject areas considered for rejection was screened. By assuming that the subject areas' top-20 most cited articles represent the subject areas' relevance, a subject area was only included if the relevant of the top-20 most cited papers were not present in already included subject areas. This effort was conducted based on the subject area distribution from executing search string 1 (Figure 1).

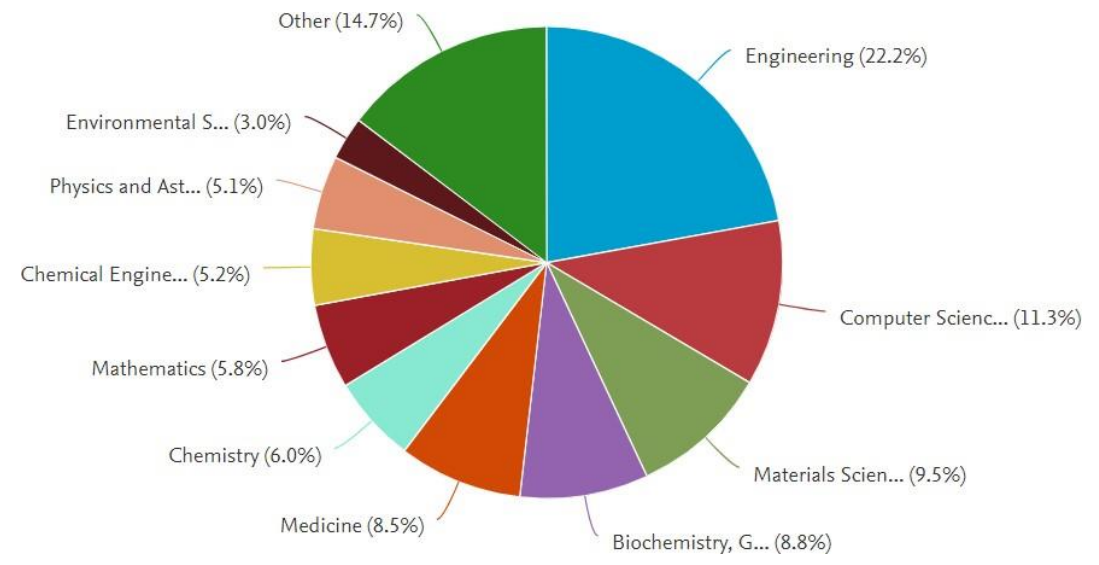

Figure 1. The distribution of articles filtered with respect to subject area, found by executing search string 1 in Scopus

Of the top-20 most cited articles within the subject area, 'Biochemistry, Genetics and Molecular Biology' - a subject area considered for rejection - three contributions by (Vincent et al., 2006), (Liu and Jiang, 2011a) and (Bar-Cohen, 2006) was assessed due to their relevancy. This eventually resulted in the rejection of this subject area, as these contributions was also found in the subject area, 'Engineering'. Next, filtering like this reduced the amount of subject areas from 28 to 14. Finally, as the ICED conference is a Design Society-conference, the electronic database of the Design Society was investigated for relevant literature, by executing the search string displayed underneath: 
"Bio-inspired design" OR "Biologically-inspired design" OR "Bio-inspired engineering" OR biomimetics OR biomimicry OR "Biological phenomen*" OR "Biological principle*" OR "Biological solution*" OR "Biological analog*" OR bionics OR biotriz.

\subsection{Systematic literature review analysis}

Finally, having increased the relevance of the found literature in a total pool of 1.446 articles, the articles found from executing the systematic literature review was now analysed with respect to the fulfilment of the inclusion criteria (proposing/reviewing tool(s) or methodologies(s) for BID) and qualification criteria (published in journals or conference proceedings) respectively.

\section{RESULTS}

Screening titles and/or abstracts of the 1.446 found articles by executing the four search strings in Scopus and Design Society's electronic database respectively, 81 articles was still relevant. Reading full paper of the 81 articles and checking interesting references, review or proposal of tool(s) or methodologies was discovered in 18 articles. Multi-functionality was addressed in 5 articles.

\subsection{Methodology supporting biologically inspired design}

The revealed methodologies and their phase-specific tools are classified with respect to 9 tasks inspired by the logical pattern developed by Paris Tech (Fayemi et al., 2014) and the design model proposed by the DTU Group (Lenau, 2018), visualized in Figure 2.

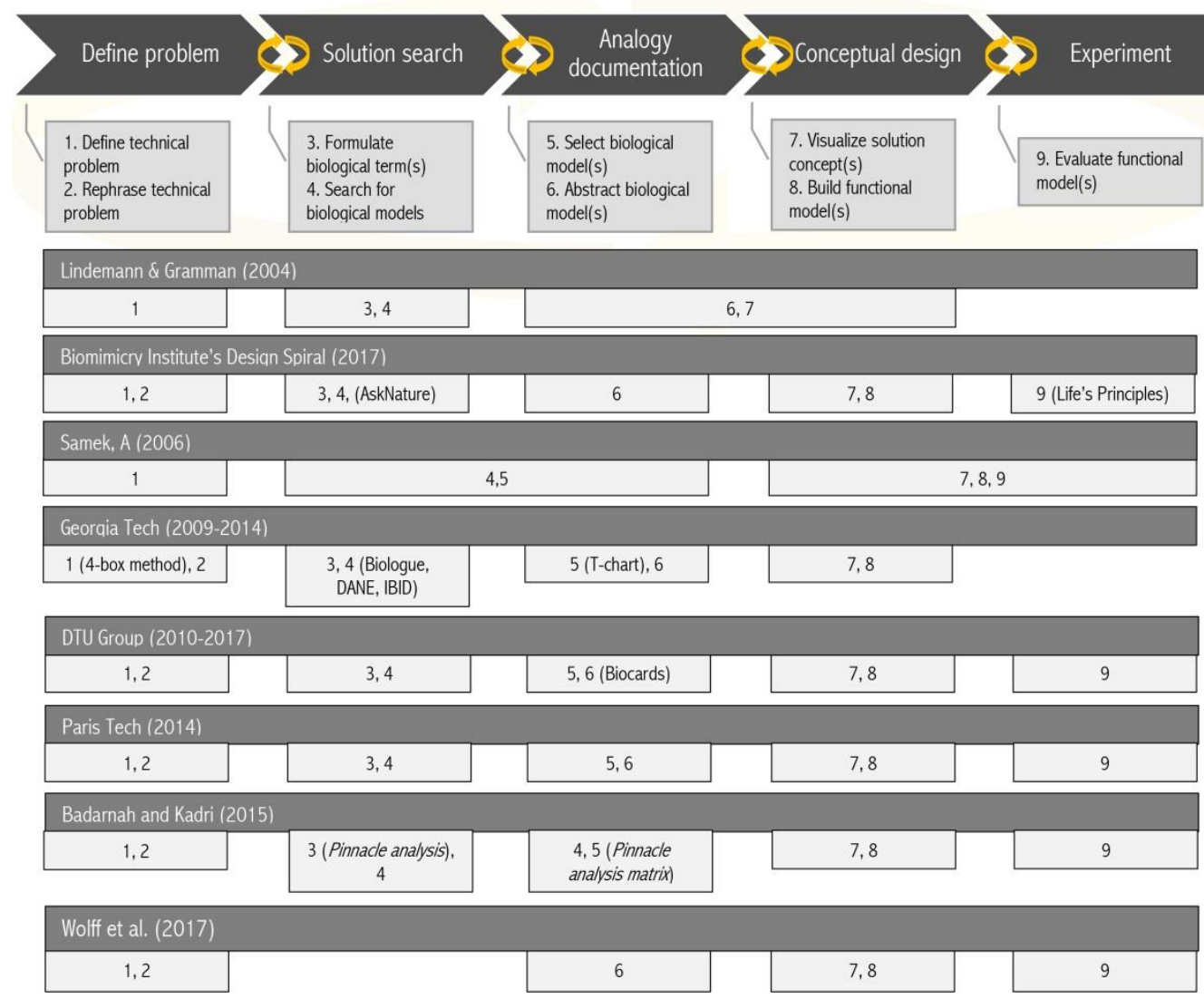

Figure 2. The discovered methodologies classified with respect to 5 phases with 9 tasks.

Tools proposed specifically for BID are noted by the default text setting while tools applicable for BID purposes are highlighted with italic

As displayed in Figure 2, not all methodologies describe all 9 tasks, e.g. the methodology from Wolff et al. don't described task 3 and 4. The systematic literature review revealed 8 BID-methodologies of which 4 provided phase-specific tool(s). The 'solution search'-phase are by far the most supported phase of BID, in particular the task 'search for biological' with 4 BID tools supporting this task. Contrary, the 'conceptual design'-phase are not supported with distinct BID tools, while the phases 'define problem' and 'experiment' are only supported with one distinct BID tool respectively. 
Early research of (Lindemann and Gramann, 2004) and (Samek, 2006) introduced frameworks for BID, though not providing detailed guidance to many phases and neither any phase-specific tools. They refer to BID as 'bionics', but that term has since then changed its meaning as it can be seen from the ISO- standard described above. The Biomimicry Institute applies a methodology designated the biomimicry spiral (DeLuca, 2017) with six phases, including support for the search phase (4) by the means of the biomimicry taxonomy and the AskNature-database providing inspirational descriptions of a wide range of biological principles and Life's Principles for the evaluation phase (9).

The Georgia Tech Group has developed a methodology for biologically-inspired design (Helms, Vattam and Goel, 2009) with addition of phase-specific support to designers. On one hand, they developed the 4-box method for problem formulation and the T-chart tool for analogy evaluation (Helms and Goel, 2014). On the other hand, an automated search engine called DANE (Vattam et al., 2010), the Biologue software aiding designers in semantically tagging biological literature (Vattam and Goel, 2013) and the intelligent search mechanism IBID (Spiliopoulou et al., 2015) was conceptualized.

The DTU group provide a methodology for biologically-inspired design covering all 5 phases of the BID process (Lenau et al., 2010) including phase-specific support for the abstraction task (6) by the means of biocards (Lenau, 2017).

Additionally, methodology without phase-specific support have been developed for BID. For instance, Paris Tech suggest a problem-driven biomimetic methodology inspired by TRIZ. It hypothesizes that the tools of TRIZ might be useful in phase 2, 3, 6 and 7 for abstraction and transfer activities respectively (Fayemi et al., 2014). In a TU Delft/MIT-collaboration, a methodology for generation of biomimetic design concepts has been developed with support for phases 3 and 5 driven by pinnacle analysis, although not support specifically targeted at BID (Badarnah and Kadri, 2015) . In another cross- institutional collaboration, the Macquaire University and the University of New South Wales, has developed a 5 step methodology for enhanced outcome of biomimetic programs (Wolff et al., 2017).

However, tools addressing single-phase activities of other research groups than those proposing a methodology for BID, have been discovered. Phases 2-3 has been supported by University of Toronto developing an approach to bridge cross-domain terminology (BCDT) of engineering and biology (Chiu and Shu, 2005) and by researchers from Oregon State University and Texas A\&M developing an engineering-to-biology-thesaurus (ETBT) for engineering design (Nagel, Stone and Mcadams, 2010) - supporting the generation of keyword and terms by engineers novice to biology. The search phase (4) has been supported by the works of the University of Bath developing a contradiction matrix yielding biologically-inspired recommendations called BioTRIZ (Vincent et al., 2006), Chakrabati et al providing the IDEA-INSPIRE software for database-guided analogical search (Chakrabarti et al., 2006). Summarizing, a total of 8 methodologies in addition to 14 tools supporting the practice of different BID tasks was found by systematically reviewing state-of-the-art BID literature:

- $\quad$ Of the 8 methodologies found, 3 of them provide phase-specific BID tools for one or more phase

- No phase-specific BID tools was found for the 'conceptual design'-phase

- 1 BID tool for each of the tasks 1, 5, 6 and 9 was discovered

- 2 BID tools for each of the tasks 2 and 3 was discovered

- 6 BID tools for task 4 was discovered

Thereby, almost $50 \%$ of the discovered tools supporting BID aimed at the phase dealing with retrieving biological analogies, whereas the support for the latest phases are highly limited.

\subsection{Current state of multi-functionality in BID literature}

By systematically reviewing state-of-the-art methodologies and tools for BID, contributions was primarily found from a research group at Georgia Tech, a research group from University of Bath and a research project from Macquaire University and University of South Wales.

Assessing the discovered literature, the task of handling multi-functionality in problem-driven BID are elaborated by researchers from Macquaire University and University of South Wales: "if the aim is multi-functionality as in gecko-inspired adhesives and synthetic spider silks, then a more directed approach is to break down the problem into single, clearly defined sub-topics. Sub-topics represent single functions that are, at first, studied separately, and later jointly implemented into the product." (Wolff et al., 2017). 
The Georgia Tech Group follows a similar approach to problem-driven BID. This effort is initiated by functional decomposition followed by, a number of parallel solutions searches corresponding to the number of discovered sub functions. Subsequently, the sub functional biological analogies are merged into one solution concept. The approach to multi-functional problem driven BID is referred to as compound analogical design (Vattam, Helms and Goel, 2008). The approach was found useful from an analysis of a range of solution concepts developed at the introductory biologically-inspired engineering design course at Georgia Tech. The functional decomposition, parallel solution search and merging of bio-inspired sub functional solution principles, was found particularly useful when designers were facing projects with competing demands (Helms, Vattam and Goel, 2009).

The BioTRIZ matrix developed by researchers from The University of Bath provides support for handling multi-functionality in BID. The support primarily address the 'problem definition'-phase of the BID process. In TRIZ, the functional problem is rephrased to form a contradiction, e.g. parameter 1 should be optimized/kept stable (thesis) while parameter 2 should be kept stable/limited (antithesis). Next, in the contradiction matrix, the thesis is matched with one of the 39 contradiction features of the vertical axis while the antithesis is matched with one of 39 contradiction features on horizontal axis. Last, inventive principles are discovered in the cell of the matrix corresponding to the chosen thesis row and antithesis column. From an analysis of 2500 contradictions and their biological resolutions, BioTRIZ constitute the condensed contradiction matrix, only proposing inventive principles that could aid a BID development project (Vincent et al., 2006). To display how TRIZ support multifunctionality, consider the development of endoscopic surgical equipment. For this case, surgery time should be limited (contradiction feature to optimize). Simultaneously, at the end of the endoscope the camera is placed, whereby the cross-sectional area of the endoscope is fixed (contradiction feature to preserve). Using the TRIZ contradiction matrix and choosing 'speed' for the optimized feature and 'shape' for the preserved feature, inventive principle 15, 18, 34 and 35 proposed (SolidCreativity, 2014). As the inventive principles are based on already existing patents and inventions, this contradiction has been resolved by developing flexible endoscopes (inventive principle 15). Likewise, for a similar problem, a wasp-inspired steerable needle has been developed at the Bio-Inspired technology Group at TU Delft (Scali et al., 2017).

The Georgia Tech Group has also assessed multi-functionality in solution-driven BID by analysing the final designs of students participating in an introductory course on biologically-inspired engineering design (Helms, Vattam and Goel, 2009) and by analysing cases from a digital study library of biologically-inspired design cases (Goel et al., 2016). Both research efforts pointed towards the fact that, solution-driven BID results in more multi-functional biologically-inspired designs than problemdriven BID. This was quantitatively determined in the 2016-study, where the $81 \%$ of the solutiondriven BID projects had multi-functional solution concepts, contrary to the problem-driven BID projects, where only $30 \%$ of the solution concepts were multi-functional.

The present study only found a limited amount of research within the area of multi-functional BID. In particular, the research area of dealing with multi-functional problems in problem-driven BID without subsequent super positioning of sub functional biological analogies - constitute a knowledge gap in existing BID theory.

\section{DIscussion}

The results and methods applied to discover state-of-the-art methodologies and tools for BID (1) and the current state of multi-functionality in BID and related research (2) will be discussed in the following sections.

\subsection{Identifying state-of-the art BID with a systematic literature review}

Due to the rigorously followed research approach of the systematic literature review, the research work is easily repeatable. In addition, the systematic literature review appears comprehensible, reflecting on the number of keywords in every search block and the number of screened papers. The risk of unintentionally omitting potentially relevant contributions are avoided by including a wide range of subject areas (displayed in Figure 2).

However, while the thoroughness of a systematic literature review might be a benefit in this research work, simultaneously it has possibly increased the review task. Retrieving more than 1.400 relevant from executing the 3 search strings configured from the 4 search blocks (section 2.1) and ending up 
with a pool of 81 papers yields an inclusion percentage only around 5\%. In this regard, a "roll the snowball"-research approach similar to that of the cross-institutional collaboration effort of Deggendorf Institute of Technology, Paris Tech and TU Munich (Wanieck et al., 2017), could have been applied. Although, this research approach would be limited by collaboration partners while risking the missing of relevant contributions from adjacent research fields. Thus, the systematic review of literature identifying state- of-the-art methodologies and tools for BID practice and the current state of multi-functionality in BID has both benefits and disadvantages. Nevertheless, due to the limited research experience and network size of the first author, an expert within BID was consulted prior to the formulation of keywords was initiated, and the systematic literature review-approach was chosen.

\subsection{The current state of multi-functionality in BID and related research}

Research and experiments dealing with multi-functionality in BID was found both within solutionand problem-driven BID, with most actual support for the problem-driven approaches. While the BioTRIZ matrix primarily aid the initial phases of the BID process, the compound analogical design approach aid both the problem definition and the conceptual design of the BID process, displayed in Figure 3.

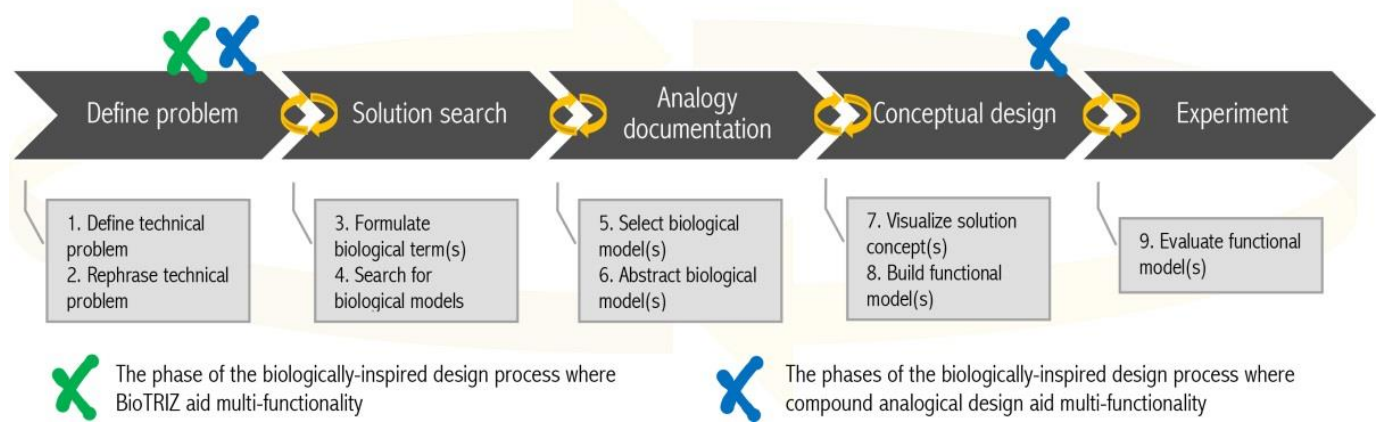

Figure 3. Overview of where in the BID design process existing BID approaches provides aid for problem-driven multifunctional BID

In continuation of this, it is hypothesized that, retrieving multi-functional biological analogies will improve efficiency of the design process by easing or eliminating the need for integrating sub functional bioinspired solution concepts. This motivation is underlined by the Georgia Tech group recommending looking for organisms with single solutions solving multiple problems simultaneously as one of their solution search techniques (Helms, Vattam and Goel, 2009). Furthermore, this motivation is also justified in an assay from the National Centre for Biological Sciences, recommending that engineers focus on the multi-functionality of biological organism (Sane, 2016). While, neither Helms, Vattam and Goel nor Sane explicate the benefits of multi-functional analogies, it's the authors impression that improving efficiency constitute the main reasoning. This benefit is explicated in other research pointing towards the fact that multi-functional designs have a characteristic of being energy- and materials efficient by default. This is, a product with parts performing multiple functions is likely due to be constituted by fewer parts and thereby reducing environmental effects by requiring less material and being lighter (O'Rourke \& Seepersad, 2015).

However, while the compound analogical design approach might foster bio-inspired solutions, conducting several parallel BID efforts corresponding to every sub function limits the efficiency of the design process. Furthermore, like outlined by the Georgia Tech research group (Goel, Hancock, Fraser, \& Tuchez, 2016), the whole concept of the existing problem-driven BID approaches implying functional decompositioning is counterintuitive in terms of providing multi-functional analogies. This suggests that the gap to be addressed by this research going forward is situated in the two initial phases of the BID process - where the problem is defined and analogies are discovered. Last, by juxtaposing the existing procedures aiding multifunctionality in BID (Figure 3), the incentives of discovering multi-functional analogies and the findings of Goel et al. regarding multi-functionality in solutiondriven BID, supporting designers in converting a problem-driven BID process to a solution-driven BID process in the early phases of the BID process will be the ambition of this research going forward. 
The topic of handling multi-functionality in product development are also of interest in adjacent research fields. For instance, materials science researchers from Beihang University has reviewed a wide range of bio-inspired materials performing multiple functions (Liu and Jiang, 2011b).

Likewise, chemical researchers from a Dalian University of Technology/McMasters Universitycollaboration have developed a paper-based sensor with bio/nano materials respectively sensing molecular presence and signal transmittance (Hui et al., 2018). Both research approaches, documenting approaches somewhat similar to the solution-driven BID approach. Thereby, future development of design support for multi-functionality in BID will possibly benefit from screening adjacent research fields' approaches to multi-functionality.

Considering, the case of developing an anterior eye-chamber model, one could apply a problem-driven multi-functional BID approach or consult a biologist to carry out a solution-driven BID approach to replicate the structures of the human eye to provide a similar functionality. However, as a biological model, the human eye is constituted by structures providing multiple different functions reaching far beyond ensuring the life of the cornea. Thereby, for the purpose of this project, such an approach will be unnecessarily complicated while not necessarily yielding a satisfactory result within the time frame of a $\mathrm{PhD}$ project while providing design support for biologists and not engineers. Therefore, the problem-driven BID approach to handle multi-functionality will be explored going further.

\section{CONCLUSION}

The case example describing the development of an anterior eye-chamber model pointed towards the fact that existing BID methodologies and phase-specific tools for BID are misaligned with the designers' need. Current single-function focus of BID methodologies is misaligned with the fact that products performing multiple functions are often demanded. Thus, the present approach to multifunctional problems, implying parallel BID efforts for every function, is time-consuming as a number of bio-inspired solution concepts corresponding to the number of functions of a product must be discovered. Likewise, this approach is also inefficient as new sub functional requirements are likely to arise as a result of the combination of the bio-inspired partial solutions. Contrary, the contributions discovered and assessed useful for handling multi-functionality in BID, points towards focusing on supporting the early phases of the BID process in transitioning from problem-driven BID to solutiondriven BID. Thus, reducing the extent of the search phase and simplifying the bio-inspired solution integration task in the conceptual design phase could be achieved.

This research facilitated the assessment of the current state of multi-functionality in state-of-the-art BID theory. Through the systematic literature review, 18 articles describing 8 BID methodologies and 14 BID tools in addition to 9 articles addressing multi-functionality in BID was discovered from a pool of 81 articles, satisfying both criteria of inclusion and qualification. The discovered BID methodologies and tools for BID were categorized with respect to 5 generic phases and 9 tasks of problem-driven BID adapted from works of BID groups from DTU and Paris Tech. This categorization displayed that almost 50\% of the discovered BID tools addressed the 'solution search'phase, suggesting that this is the most difficult phase of problem-driven BID using existing methodologies for BID, which all are guided by single-function problems.

Therefore, we suggest expanding the BID focus to multi-functional BID also, in order to develop useful design support complying with the needs of designers.

\section{REFERENCES}

Badarnah, L. and Kadri, U. (2015), "A methodology for the generation of biomimetic design concepts", Architectural Science Review, Vol. 58 No. 2, pp. 120-133. 10.1080/00038628.2014.922458.

Bar-Cohen, Y. (2006), "Biomimetics - Using nature to inspire human innovation", Bioinspiration and Biomimetics, Vol. 1 No. 1. 10.1088/1748-3182/1/1/P01.

Chakrabarti, A. et al. (2006), "A functional representation for aiding biomimetic and artificial inspiration of new ideas”, Artificial Intelligence for Engineering Design, Analysis and Manufacturing: AIEDAM, Vol. 19 No. 2 , pp. 113-132. 10.1017/S0890060405050109.

Chiu, I. and Shu, L. (2005), "Bridging cross-domain terminology for biomimetic design", Proceedings of 2005 ASME International Design Engineering Technical Conferences and Computers and Information in Engineering Conference, Long Beach, California, USA, pp. 1-9. 10.1115/DETC2005-84908.

DeLuca, D. (2017), "The power of the Biomimicry Design Spiral”, Biomimicry Inst., https://biomimicry.org/biomimicry-design-spiral/ 
Fayemi, P. E. et al. (2014), "Bio-inspired design characterisation and its links with problem solving tools", Proceedings of International Design Conference, DESIGN, 2014-January (December 1960), pp. 173-182.

Goel, A. et al. (2016), "Does the Biologically Inspired Design Process Result in More Multifunctional Designs ?", (4th ICDC), pp. 1-8.

Graeff, E., Maranzana, N. and Aoussat, A. (2018), "Role of biologists in biomimetic design processes : preliminary results”, International Design Conference, pp. 1149-1160. 10.21278/idc.2018.0426.

Hashemi Farzaneh, H. (2016), "Bio-inspired design: Ideation in collaboration between mechanical engineers and biologists", p. 279.

Helms, M. and Goel, A. K. (2014), "The Four-Box Method: Problem Formulation and Analogy Evaluation in Biologically Inspired Design”, Journal of Mechanical Design, Vol. 136 No. 11, p. 111106. 10.1115/1.4028172.

Helms, M., Vattam, S. S. and Goel, A. K. (2009), "Biologically inspired design: process and products", Design Studies. Elsevier Ltd, Vol. 30 No. 5, pp. 606-622. 10.1016/j.destud.2009.04.003.

Hui, C. Y. et al. (2018), “A Paper Sensor Printed with Multifunctional Bio/Nano Materials”, Angewandte Chemie, Vol. 130, pp. 4639-4643. 10.1002/ange.201712903.

ISO 18458 (2015), "Biomimetics - Terminology, concepts and methodology”. 10.1021/es0620181.

Kennedy, B., Buikema, A. and Nagel, J. K. S. (2015), "Integrating Biology, Design, and Engineering for Sustainable Innovation”, 2015 IEEE Integrated STEM Education Conference. IEEE, pp. 88-93. 10.1109/ISECon.2015.7119952.

Lenau, T. A. (2009), "Biomimetics as a design methodology - Possibilities and challenges”, International conference on engineering design, (August), pp. 121-132.

Lenau, T. A. et al. (2010), "Engineering design of an adaptive leg prosthesis using biological principles", 11th International Design Conference (design 2010), pp. 331-340.

Lenau, T. A. (2017), “Do Biomimetic Students Think Outside the Box?”, Vol. 4 No. 21, pp. 543-551.

Lenau, T. A. (2018), "Paradigms for biologically inspired design”. 10.1117/12.2296560.

Lindemann, U. and Gramann, J. (2004), "Engineering design using biological principles", International Design Conference, pp. 355-360.

Liu, K. and Jiang, L. (2011a) "Bio-inspired design of multiscale structures for function integration", Nano Today. Elsevier Ltd, Vol. 6 No. 2, pp. 155-175. 10.1016/j.nantod.2011.02.002.

Liu, K. and Jiang, L. (2011b) "Multifunctional Integration: From Biological to Bio-Inspired Materials", Vol. 5 No. 9, pp. 6786-6790. 10.1021/nn203250y.

Nagel, J. K. S., Stone, R. B. and Mcadams, D. A. (2010), “An Engineering-To-Biology Thesaurus for Engineering Design”, ASME 2010 International Design Engineering Technical Conference \& Computers and INformation in Engineering Conference, (October), pp. 1-11. 10.1115/DETC2010-28233.

Neinhuis, C. and Barthlott, W. (1997), "Characterization and Distribution of Water-repellent, Self-cleaning Plant Surfaces", pp. 667-677.

Samek, A. (2006), "BIONICS IN THE DESIGNING”, General approaches to the design process, pp. 129-135.

Sane, S. P. (2016), "Bioinspiration and biomimicry: What can engineers learn from biologists?”, Journal of Applied Science and Engineering, Vol. 19 No. 1, pp. 1-6. 10.6180/jase.2016.19.1.01.

Scali, M. et al. (2017), "Design and evaluation of a wasp-inspired steerable needle", Bioinspiration, Biomimetics, and Bioreplication. 10.1117/12.2259978.

SolidCreativity (2014), “Triz40 [online] SolidCreativity.” Available at http://www.triz40.com/TRIZ_GB.php (10 December 2018).

Spiliopoulou, E. et al. (2015), "Intelligent Search for Biologically Inspired Design", Proceedings of the 20th International Conference on Intelligent User Interfaces Companion, pp. 77-80. 10.1145/2732158.2732182.

Tero, A. et al. (2010), "Rules for biologically inspired adaptive network design", Science, Vol. 327 No. 5964, pp. 439-442. 10.1126/science.1177894.

Vattam, S. et al. (2010), "DANE: Fostering creativity in and through biologically-inspired design”, Vol. 8 No. November, pp. 115-122.

Vattam, S. S. and Goel, A. K. (2013), "Biological solutions for engineering problems: A study in cross-domain textual case-based reasoning", Lecture Notes in Computer Science (including subseries Lecture Notes in Artificial Intelligence and Lecture Notes in Bioinformatics), Vol. 7969 LNAI(2001), pp. 343-357. 10.1007/978-3-642-39056-2 25.

Vattam, S. S., Helms, M. E. and Goel, A. K. (2008), "Compound Analogical Design: Interaction between Problem Decomposition and Analogical Transfer in Biologically Inspired Design”, Design Computing and Cognition '08, pp. 377-396. 10.1007/978-1-4020-8728-8_20.

Vincent, J. F. V. et al. (2006), "Biomimetics: its practice and theory", Journal of The Royal Society Interface, Vol. 3 No. 9, pp. 471-482. 10.1098/rsif.2006.0127.

Wanieck, K. et al. (2017), "Biomimetics and its tools", Bioinspired, Biomimetic and Nanobiomaterials, Vol. 6 No. 2, pp. 53-66. 10.1680/jbibn.16.00010.

Wolff, J. O. et al. (2017), "Clarity of objectives and working principles enhances the success of biomimetic programs", Bioinspiration and Biomimetics. IOP Publishing, Vol. 12 No. 5. 10.1088/1748-3190/aa86ff. 